\title{
GEOMETRY AND PRODUCT STATES
}

\author{
Robert B.Lockhart ${ }^{1}$, Michael J. Steiner ${ }^{2}$, Karl Gerlach ${ }^{2}$ \\ 1 Mathematics Department, United States Naval Academy, Annapolis, Maryland 21401 \\ 2 Naval Research Laboratory, Washington D.C.
}

\begin{abstract}
As separable states are a convex combination of product states, the geometry of the manifold of product states, $\Sigma$ is studied. Prior results by Sanpera, Vidal and Tarrach are extended. Furthermore, it is proven that states in the set tangent to $\Sigma$ at the maximally mixed state are separable; the set normal constains, among others, all maximally entangled states. A canonical decomposition is given. A surprising result is that for the case of two particles, the closest product state to the maximally entangled state is the maximally mixed state. An algorithm is provided to find the closest product state.
\end{abstract}

PACS numbers: $03.67,03.65 . \mathrm{Bz}$

\section{INTRODUCTION}

Entanglement has always been one of the quantum phenomena which sparked debates about the completeness and interpretations of quantum mechanics [1], [2], [3]. It is fundamental to teleportation [4], [5], secure key distribution, [6], [7], dense coding [5] and other applications.

Obviously, therefore, properties of entangled states, including ways to determine if a state is entangled, are important. With regard to the latter, several authors have given sufficient, necessary, and necessary and sufficient conditions for a state to be entangled [8], [9], [10], 
[11]. With the exception of Peres's partial transpose condition - if any partial transpose of a state has a negative eigenvalue, then the state is entangled - these conditions tend to be either impossible or hard to implement in all but low dimensional, low rank cases. This, of course, is an important starting point for the investigation of entanglement.

In this paper we take a different approach. Rather than trying to determine if a state is entangled or not - the latter case being known as separable - we study the whole set of separable states and the whole set of entangled ones. Our approach is geometric and starts with the manifold of product states, which we denote by $\Sigma$. One reason for starting with $\Sigma$ is that it is easy to decide if a density matrix is a product state by using partial trace. Another reason is that the totally mixed state $\frac{1}{N} I$ is a product state. Lastly, $\Sigma$ is of interest in itself. It is not convex or linear. But, as we shall show, it does have a nice geometric property: if a straight line intersects $\Sigma$ in more than two states, then every point on the line is a product state. Sanpera, Vidal and Tarrach [12] proved this in the case of two qubits. We show that it is true in general.

After establishing this geometric result, we turn to examining the set of entangled and the set of separable states. Our approach is to start at the totally mixed state, $\frac{1}{N} I$ and go in various directions. For instance, we show that all states which are in the set tangent to $\Sigma$ at $\frac{1}{N} I$ are separable. On the other hand the maximally entangled states are in the set which is normal to $\Sigma$ at $\frac{1}{N} I$.

One of our main results is the fact that in the case of $\mathbb{C}^{n} \otimes \mathbb{C}^{n}$ the product state which is closest to a maximally entangled state is $\frac{1}{N} I$, the totally mixed state. This surprising result, which we shall, unfortunately, show is false for systems with more than two particles, has an important consequence: there are no product states inside the ball of radius $\sqrt{\frac{N-1}{N}}$ centered at a maximally entangled state.

It may seem to the reader that it would be hard to determine if a state is in the sets tangent or normal to $\Sigma$ at $\frac{1}{N} I$ and so the results just mentioned may seem to be hard to use. However, this is not the case. In fact it is easy to determine if a state is in the tangent or normal set. One uses a canonical, orthogonal decomposition of $\tau_{0}(N)$, the vector space of 
trace 0 , Hermitian, $N \times N$ matrices, and the fact that every density matrix, $Q$, is uniquely expressible as $Q=\frac{1}{N} I+H$, where $H \in \tau_{0}(N)$.

This fact and the canonical decomposition of $\tau_{0}(N)$ are fairly standard mathematical fare; perhaps because $i \tau_{0}(N)$ is the Lie Algebra $s u(N)$. However, it might not be well-known in the Quantum Information Community. Therefore, we shall present it in detail for general multi-particle systems. To get an idea of what is involved, consider the case of a density matrix $Q$ on $\mathbb{C}^{n} \otimes \mathbb{C}^{m}$. It has the orthogonal decomposition $Q=\frac{1}{N} I+H_{1} \otimes \frac{1}{m} I+\frac{1}{n} I \otimes H_{2}+H_{3}$, where $H_{1} \in \tau_{0}(n), H_{2} \in \tau_{0}(m)$, and $H_{3} \in \tau_{0}(n) \otimes \tau_{0}(m)$. It follows from results in [13] that $Q$ is in the tangent space of $\Sigma$ at $\frac{1}{N} I$ if and only if $H_{3}=0$. Notice, it follows from Theorem 3 in the present paper that if $\mathrm{Q}$ is entangled, then $H_{3}$ is not zero. We see from this decomposition, therefore, conditions under which entanglement occurs. As for $Q$ being in the normal space of $\Sigma$ at $\frac{1}{N} I$, that happens if and only if $H_{1}=H_{2}=0$. This follows, easily, from the orthogonality of the decomposition. To actually find the decomposition for a given state, one needs only to choose orthonormal bases for $\tau_{0}(n)$ and $\tau_{0}(m)$. The set of their tensor products then forms an orthonormal basis for $\tau_{0}(n) \otimes \tau_{0}(m)$. Given these three orthonormal bases, one then just takes inner products to get the decomposition.

We finish this paper with an algorithm which appears, in the bipartite case, to give the product state which is closest to a given one.

\section{PRODUCT STATES}

We first establish some useful notation:

$$
\tau_{k}(N)=\left\{A \mid A \text { is a Hermitian operator on } \mathbb{C}^{N} \text { and } \operatorname{Tr}(A)=k\right\}
$$

and

$$
\mathcal{D} \mathcal{M}(N)=\left\{A \mid A \text { is a density matrix on } \mathbb{C}^{N}\right\},
$$

where $\operatorname{Tr}$ denotes trace. Evidently, $\mathcal{D} \mathcal{M}(N)$ is the subset of $\tau_{1}(N)$ consisting of positive, semi-definite operators. For $N=n_{1} \cdots n_{p}$, we need to describe the set of product state 
density matrices on $\mathbb{C}^{N}=\mathbb{C}^{n_{1}} \otimes \cdots \otimes \mathbb{C}^{n_{p}}$. Using the notation from [13], we have the product operator

$$
\begin{aligned}
\mu: \Pi_{1}^{p} \tau_{1}\left(n_{i}\right) & \rightarrow \tau_{1}\left(\Pi_{1}^{p} n_{i}\right)=\tau_{1}(N), \quad \text { given by } \\
\mu\left(A_{1}, \ldots, A_{p}\right) & =A_{1} \otimes \cdots \otimes A_{p} .
\end{aligned}
$$

The image of $\mu$ in $\tau_{1}(N)$, denoted by $\Sigma$, is a closed, embedded submanifold 13. In particular, every point in $\Sigma$ has a tangent space and normal space. We shall use this later, but first we extend the result of Sanpera, Tarrach and Vidal [12] about lines. Before doing so, we must point out that the set of product density matrices, denoted $\Sigma^{+}$, in $\mathcal{D} \mathcal{M}(N)$ is just the image of $\mu$ restricted to $\Pi_{1}^{p} \mathcal{D} \mathcal{M}\left(n_{i}\right)$ and so is a subset of $\Sigma$. Thus the next theorem holds a fortiori for product density matrices.

Theorem 1 If $A_{1} \otimes \cdots \otimes A_{p}=A$ and $B \in \tau_{1}(N)$, then one of the following is true about the line $r(t)=t\left(A_{1} \otimes \cdots \otimes A_{p}\right)+(1-t) B$ :

1. It intersects $\Sigma$ only at $A_{1} \otimes \cdots \otimes A_{p}$,

2. It intersects $\Sigma$ at $A_{1} \otimes \cdots \otimes A_{p}$ and exactly one other point,

3. The line lies in $\Sigma$. In particular every density matrix on the line is a product state.

Proof:

Suppose $B \neq A$ is also a product, thus $B=B_{1} \otimes \cdots \otimes B_{p}$, and suppose $t_{0} A_{1} \otimes \cdots \otimes A_{p}+$ $\left(1-t_{0}\right) B_{1} \otimes \cdots \otimes B_{p}=C_{1} \otimes \cdots \otimes C_{p}$ for some $t_{0} \neq 0$ or 1 . In other words, suppose there are three products on the line. We need to show $r(t)=t A_{1} \otimes \cdots \otimes A_{p}+(1-t) B_{1} \otimes \cdots \otimes B_{p}$ is a product for all t. If $A_{2} \otimes \cdots \otimes A_{p}=B_{2} \otimes \cdots \otimes B_{p}$, then we have $r(t)=\left(t A_{1}+(1-t) B_{1}\right) \otimes$ $A_{2} \otimes \cdots \otimes A_{p}$ and so are done. Therefore assume $A_{2} \otimes \cdots \otimes A_{p} \neq B_{2} \otimes \cdots \otimes B_{p}$. Note, since $\operatorname{Tr}\left(A_{2} \otimes \cdots \otimes A_{p}\right)=1=\operatorname{Tr}\left(B_{2} \otimes \cdots \otimes B_{p}\right)$, we have that $A_{2} \otimes \cdots \otimes A_{p} \neq B_{2} \otimes \cdots \otimes B_{p}$ is equivalent to them being linearly independent.

Let $\left\{E_{i}\right\}$ be a basis for $\tau_{1}\left(n_{i}\right)$. Then, using the Einstein summation convention of summing over repeated upper and lower indices, we have 


$$
t_{0} A_{1}^{i} E_{i} \otimes A_{2} \otimes \cdots \otimes A_{p}+\left(1-t_{0}\right) B_{1}^{i} E_{i} \otimes B_{2} \otimes \cdots \otimes B_{p}=C_{1}^{i} E_{i} \otimes C_{2} \otimes \cdots \otimes C_{p} .
$$

Since the $E_{i}$ are linearly independent, this means that for each $i$

$$
t_{0} A_{1}^{i} A_{2} \otimes \cdots \otimes A_{p}+\left(1-t_{0}\right) B_{1}^{i} B_{2} \otimes \cdots \otimes B_{p}=C_{1}^{i} C_{2} \otimes \cdots \otimes C_{p}
$$

If $C_{1}^{i_{0}}=0$ for some $i_{0}$, then $A_{1}^{i_{0}}=0=B_{1}^{i_{0}}$. For otherwise we would have $t_{0} A_{1}^{i_{0}} A_{2} \otimes \cdots \otimes$ $A_{p}+\left(1-t_{0}\right) B_{1}^{i_{0}} B_{2} \otimes \cdots \otimes B_{p}=0$, which would contradict our assumption that $A_{2} \otimes \cdots \otimes A_{p}$ and $B_{2} \otimes \cdots \otimes B_{p}$ are linearly independent. Therefore assume $C_{1}^{i_{1}} \neq 0$ in which case we get

$$
\frac{t_{0} A_{1}^{i_{1}}}{C_{1}^{i_{1}}} A_{2} \otimes \cdots \otimes A_{p}+\frac{\left(1-t_{0}\right) B_{1}^{i_{1}}}{C_{1}^{i_{1}}} B_{2} \otimes \cdots \otimes B_{p}=C_{2} \otimes \cdots \otimes C_{p} .
$$

It follows from this and the linear independence of $A_{2} \otimes \cdots \otimes A_{p}$ and $B_{2} \otimes \cdots \otimes B_{p}$ that $\frac{t_{0} A_{1}^{i_{1}}}{C_{1}^{i_{1}}}=\frac{t_{0} A_{1}^{i_{2}}}{C_{1}^{i_{2}}}$ and $\frac{\left(1-t_{0}\right) B_{1}^{i_{1}}}{C_{1}^{i_{1}}}=\frac{\left(1-t_{0}\right) B_{1}^{i_{2}}}{C_{1}^{i_{2}}}$ for every $i_{1}, i_{2}$ such that $C_{1}^{i_{1}}$ and $C_{1}^{i_{2}}$ are not 0.

Since we already know that $A_{1}^{i}=B_{1}^{i}=0$ if $C_{1}^{i}=0$, we may conclude for all $i$ that $A_{1}^{i}=$ $\frac{A_{1}^{i_{1}}}{C_{1}^{i_{1}}} C_{1}^{i}$ and $B_{1}^{i}=\frac{B_{1}^{i_{1}}}{C_{1}^{i_{1}}} C_{1}^{i}$, where $C_{1}^{i_{1}} \neq 0$. Thus $A_{1}=\frac{A_{1}^{i_{1}}}{C_{1}^{i_{1}}} C_{1}=\lambda C_{1}$ and $B_{1}=\frac{B_{1}^{i_{1}}}{C_{1}^{i_{1}}} C_{1}=\mu C_{1}$. Since $\operatorname{Tr}\left(A_{1}\right)=\operatorname{Tr}\left(B_{1}\right)=\operatorname{Tr}\left(C_{1}\right)=1$, we have $\lambda=\mu=1$ and so $A_{1}=B_{1}=C_{1}$. This means we may write

$$
t_{0} A_{1} \otimes \cdots \otimes A_{p}+\left(1-t_{0}\right) B_{1} \otimes \cdots \otimes B_{p}=C_{1} \otimes \cdots \otimes C_{p}
$$

as

$$
A_{1} \otimes\left(t_{0} A_{2} \otimes \cdots \otimes A_{p}+(1-t) B_{2} \otimes \cdots \otimes B_{p}\right)=A_{1} \otimes C_{2} \otimes \cdots \otimes C_{p}
$$

By induction we have that $r(t)=t A_{1} \otimes \cdots \otimes A_{p}+(1-t) B_{1} \otimes \cdots \otimes B_{p}$ is a product for all $\mathrm{t}$. Hence if there are three matrices on the line $r(t)$ which are products, then all are products.

This actually proves the theorem, though one wants to know if the other two cases (one product or two products) can occur. In the next theorem we show that there are lines with only one product matrix on them. As for there being lines with only two, if that did not occur then every two elements in $\Sigma$ would be connected by a line in $\Sigma$. This would mean $\Sigma$ is convex. In particular every separable state would be a product state. However, this cannot be, for it was shown in [13] that $\Sigma^{+}$has measure 0 in $\mathcal{D} \mathcal{M}(N)$; but the set of separable 
states has an open interior and so is not measure 0 . Thus there are lines with exactly two product states on them

Before proceeding, we need to say a few words about the set of vectors tangent to $\Sigma$. Since $\Sigma$ is the image of the embedding $\mu$, it follows that the tangent space at $A=A_{1} \otimes \cdots \otimes A_{p}$ is $d \mu\left(T_{\left(A_{1}, \ldots A_{p}\right)}\right)$, where $T_{\left(A_{1}, \ldots A_{p}\right)}$ is the tangent space of $\Pi_{1}^{p} \tau_{1}\left(n_{i}\right)$ at $\left(A_{1}, \ldots A_{p}\right)$ and $d \mu$ is the Jacobian of $\mu$. Thus the tangent space of $\Sigma$ at $A=A_{1} \otimes \cdots \otimes A_{p}$ is the vector space spanned by

$$
\left\{\begin{array}{c}
H_{1} \otimes A_{2} \otimes \cdots \otimes A_{p}, A_{1} \otimes H_{2} \otimes A_{3} \otimes \cdots \otimes A_{p}, \\
\ldots, A_{1} \otimes \cdots \otimes A_{p-1} \otimes H_{p}: H_{i} \in \tau_{0}\left(n_{i}\right)
\end{array}\right\} .
$$

Note, we distinguish between the tangent space of $\Sigma$ at $A$ and the set tangent to $\Sigma$ at $A$. The former is the vector space of vectors which are tangent to $\Sigma$ at $\frac{1}{N} I$ and so provide the directions which are tangent to $\Sigma$ at $A$. The latter is the set of states which are obtained by starting at $A$ and going in a tangential direction. Thus if $\mathcal{T}_{\mathcal{A}}$ denotes the tangent space of $\Sigma$ at $A$, then the set tangent to $\Sigma$ at $A$ is $A+\mathcal{T}_{\mathcal{A}}$. Similarly, the normal space of $\Sigma$ at $A$ is the vector space of vectors normal to $\Sigma$ at $A$ and the set normal to $\Sigma$ at $A$ is the set of states which are obtained by starting at $A$ and going in a normal direction. If $\mathcal{N}_{\mathcal{A}}$ denotes the normal space, then the set normal to $\Sigma$ at $A$ is $A+\mathcal{N}_{\mathcal{A}}$.

Theorem 2 Let $A=A_{1} \otimes \cdots \otimes A_{p}$ be such that each $A_{i}=\frac{1}{n_{i}} I$ for $i \neq i_{0}$. If the line $r(t)=t R+(1-t) A$ is orthogonal to the tangent space of $\Sigma$ at $A$, then $A$ is the only product matrix on the line.

Proof:

Suppose $Q=Q_{1} \otimes \cdots \otimes Q_{p}$ is also on the line. Since the line and tangent space are orthogonal, we have for all $j$ and all $H_{j} \in \tau_{0}\left(n_{j}\right)$

$$
\begin{aligned}
0= & \left\langle Q-A, A_{1} \otimes \cdots \otimes H_{j} \otimes \cdots A_{p}\right\rangle= \\
& \left\langle Q_{1}, A_{1}\right\rangle \cdots\left\langle Q_{j}, H_{j}\right\rangle \cdots\left\langle Q_{p}, A_{p}\right\rangle-\left\langle A_{1}, A_{1}\right\rangle \cdots\left\langle A_{j}, H_{j}\right\rangle \cdots\left\langle A_{p}, A_{p}\right\rangle .
\end{aligned}
$$


Now note that for $i \neq i_{0}$ we have $\left\langle Q_{i}, A_{i}\right\rangle=\left\langle Q_{i}, \frac{1}{n_{i}} I\right\rangle=\frac{1}{n_{i}} \operatorname{Tr} Q_{i}=\frac{1}{n_{i}}$, and $\left\langle A_{i}, A_{i}\right\rangle=$ $\left\langle\frac{1}{n_{i}} I, \frac{1}{n_{i}} I\right\rangle=\frac{1}{n_{i}}$. Thus when $j=i_{0}$, (5) becomes $\left\langle Q_{i_{0}}, H_{i_{0}}\right\rangle=\left\langle A_{i_{0}}, H_{i_{0}}\right\rangle$ for all $H \in$ $\tau_{0}\left(n_{i_{0}}\right)$. This can only happen if $Q_{i_{0}}=A_{i_{0}}$. However, if $j \neq i_{0}$ then (5) reduces to $\left\langle Q_{i_{0}}, A_{i_{0}}\right\rangle\left\langle Q_{j}, H_{j}\right\rangle=0$ for all $H_{j} \in \tau_{0}\left(n_{j}\right)$, since in this case $A_{j}=\frac{1}{n_{j}} I$ and $\left\langle\frac{1}{n_{j}} I, H_{j}\right\rangle=0$. Thus $\left\langle Q_{j}, H_{j}\right\rangle=0$ for all $H_{j}$ and so $Q_{j}=\frac{1}{n_{j}} I=A_{j}$ for all $j \neq i_{0}$.

We finish this section by showing every density matrix in the set tangent to $\Sigma$ at the totally mixed state is separable.

Theorem 3 Suppose $Q$ is a density matrix and $Q$ is in the set tangent to $\Sigma$ at $\frac{1}{N} I$, then $Q$ is separable.

Proof:

For $Q$ to be in the set tangent to $\Sigma$ at $\frac{1}{N} I, Q$ must be expressible as

$$
\begin{aligned}
Q= & \frac{1}{N} I+H_{1} \otimes \frac{1}{n_{2}} I \otimes \cdots \otimes \frac{1}{n_{p}} I+\frac{1}{n_{1}} I \otimes H_{2} \otimes \cdots \otimes \frac{1}{n_{p}} I+\cdots \\
& +\frac{1}{n_{1}} I \otimes \cdots \otimes \frac{1}{n_{p-1}} I \otimes H_{p} .
\end{aligned}
$$

If $\left|\psi_{i}\right\rangle$ is an eigenvector of $H_{i}$ with eigenvalue $\lambda_{i}$, then it is easy to see $\left|\psi_{1} \cdots \psi_{p}\right\rangle$ is an eigenvector of $Q$ with eigenvalue $\frac{1}{N}\left(1+\sum_{1}^{p} n_{i} \lambda_{i}\right)$, where, as above, $N=n_{1} \cdots n_{p}$. Since each $H_{i}$ has trace equal to 0 , the minimum eigenvalue of each $H_{i}$ is 0 , in which case $H_{i}=0$, or is negative. Let $\mu_{i}$ be the minimum eigenvalue of $H_{i}$ for each $i$. Then $\mu=\frac{1}{N}\left(1+\sum_{1}^{p} n_{i} \mu_{i}\right)$ is the minimum eigenvalue of $Q$. Since $Q$ is a density matrix it is positive semi-definite and so $0 \leq \mu$. This in turn implies $\frac{1}{N} I+\frac{1}{n_{1}} I \otimes \cdots \otimes H_{i} \otimes \cdots \otimes \frac{1}{n_{p}} I$ is positive semi-definite and so a density matrix. In fact, if $\mu_{i} \neq 0$, then $\frac{1}{N} I+\frac{1}{n_{1}} I \otimes \cdots \otimes F_{i} \otimes \cdots \otimes \frac{1}{n_{p}} I$, where $F_{i}=\frac{1}{n_{i}\left|\mu_{i}\right|} H_{i}$, is a density matrix, since the most negative eigenvalue of $F_{i}$ is $\frac{\mu_{i}}{n_{i}\left|\mu_{i}\right|}=-\frac{1}{n_{i}}$.

Noting that $\frac{1}{N} I+\frac{1}{n_{1}} I \otimes \cdots \otimes F_{i} \otimes \cdots \otimes \frac{1}{n_{p}} I=\frac{1}{n_{1}} I \otimes \cdots \otimes\left(\frac{1}{n_{i}} I+F_{i}\right) \otimes \cdots \otimes \frac{1}{n_{p}} I$, we see that $Q$ is separable, for it is the convex combination

$$
\left(1-\sum_{1}^{p} n_{i}\left|\mu_{i}\right|\right) \frac{1}{N} I+\sum_{1}^{p} n_{i}\left|\mu_{i}\right|\left(\frac{1}{n_{1}} I \otimes \cdots \otimes\left(\frac{1}{n_{i}} I+F_{i}\right) \otimes \cdots \otimes \frac{1}{n_{p}} I\right) .
$$




\section{A CANONICAL, ORTHOGONAL DECOMPOSITION FOR DENSITY MATRICES}

In this section we present a very useful orthogonal decomposition of trace 1 , Hermitian matrices in terms of the totally mixed state and trace 0, Hermitian matrices. To begin, note that if $Q$ is a trace 1 , Hermitian matrix on $\mathbb{C}^{N}$ then $Q-\frac{1}{N} I=S$ has trace 0 and so is in the vector space $\tau_{0}(N)$. Also note that a trace 0 matrix is orthogonal to $\frac{1}{N} I$, since $\left\langle\frac{1}{N} I, S\right\rangle=\frac{1}{N} \operatorname{Tr}(S)=0$. Thus we need to decompose $\tau_{0}(N)$, where $N=n_{1} \cdots n_{p}$, into orthogonal subspaces.

Let $\mathbb{Z}_{2}=\{0,1\}$. The product of p-copies of $\mathbb{Z}_{2}$ is denoted $\mathbb{Z}_{2}^{p}$. It is the set of all strings of length $\mathrm{p}$ of zeros and ones. For $\alpha=\left(\alpha_{1}, \ldots \alpha_{p}\right) \in \mathbb{Z}_{2}^{p}$ take $|\alpha|=\sum_{1}^{p} \alpha_{i}$. Thus $|\alpha|$ is the number of ones in $\alpha$. For $\alpha \in \mathbb{Z}_{2}^{p}$, take $\nu(\alpha)$ to be the vector space $\nu(\alpha)=\nu_{1}(\alpha) \otimes \cdots \otimes \nu_{p}(\alpha)$, where $\nu_{j}(\alpha)=\frac{1}{n_{j}} I$ if $\alpha_{j}=0$ and $\nu_{j}(\alpha)=\tau_{0}\left(n_{j}\right)$ if $\alpha_{j}=1$. With these notational conventions we have,

$$
\tau_{0}(N)=\oplus_{k=1}^{p} \oplus_{|\alpha|=k, \alpha \in \mathbb{Z}_{2}^{p}} \nu(\alpha)
$$

That this is an orthogonal decomposition of $\tau_{0}(N)$ follows from the fact that if $A=A_{1} \otimes$ $\cdots \otimes A_{p}$ and $B=B_{1} \otimes \cdots \otimes B_{p}$, then $\langle A, B\rangle=\prod_{i=1}^{p}\left\langle A_{i}, B_{i}\right\rangle$ and the fact that $\left\langle\frac{1}{N} I, H\right\rangle=0$ if $H$ is trace 0 .

As can be seen from (4) the subspace with $k=1$, forms the directions which are tangential to $\Sigma$ at $\frac{1}{N} I$. Thus $Q$ is in the set normal to $\Sigma$ at $\frac{1}{N} I$ if $Q=\frac{1}{N} I+S$ and $S \in \oplus_{2}^{k} \oplus_{|\alpha|=k} \nu(\alpha)$.

To close this section we mention that the only role trace 1 plays in this decomposition is to determine the scalar multiple of $I$. In particular, any observable can be similarly decomposed. 


\section{STATES THAT HAVE THE TOTALLY MIXED ONE AS THE BEST PRODUCT STATE APPROXIMATION}

Suppose $Q \in \tau_{1}(N)$, where $N=n_{1} \cdots n_{p}$. If $P$ is the closest point on $\Sigma$ to $Q$, then the line joining $Q$ to $P$ is perpendicular to the tangent space of $\Sigma$ at $P$. Thus the only density matrices which could have the totally mixed state as best product state approximation (i.e. have $\frac{1}{N} I$ as the closest product state) lie in the set normal to $\Sigma$ at $\frac{1}{N} I$. We showed in the last section how to characterize this set and shortly we shall show the maximally entangled states lie in it. But first we extend Theorem 3 by using the decomposition in the last section.

Set $\mathcal{T}=\oplus_{|\alpha|=1} \nu(\alpha)$ and $\mathcal{N}=\oplus_{k=2}^{p} \oplus_{|\alpha|=k} \nu(\alpha)$

Theorem 4 Every density matrix, $Q$, on $\mathbb{C}^{n_{1}} \otimes \cdots \otimes \mathbb{C}^{n_{p}}$ is uniquely expressible as $Q=$ $\frac{1}{N} I+Q_{\mathcal{T}}+Q_{\mathcal{N}}$, where $Q_{\mathcal{T}} \in \mathcal{T}$ and $Q_{\mathcal{N}} \in \mathcal{N}$. In fact this is an orthogonal decomposition of $Q$. If $Q$ is entangled, then $Q_{\mathcal{N}} \neq 0$. If the totally mixed state is the closest product state to $Q$, then $Q_{\mathcal{T}}=0$. If $Q_{\mathcal{N}}=0$, then $Q$ is in the set tangent to $\Sigma$ at $\frac{1}{N} I$. If $Q_{\mathcal{T}}=0$, then $Q$ is in the set normal to $\Sigma$ at $\frac{1}{N} I$.

Suppose $N=n^{p}$ and $\left|\psi_{i}\right\rangle$ is an orthonormal basis for $\mathbb{C}^{n}$. Modulo, $U(n) \otimes \cdots \otimes$ $U(n)$ action (i.e. local operations), the maximally entangled state associated with this orthonormal basis is the projection onto $\frac{1}{\sqrt{n}} \sum_{1}^{n}\left|\psi_{i} \ldots \psi_{\iota}\right\rangle$, with $\left|\psi_{i} \ldots \psi_{i}\right\rangle$ being the p-fold tensor product of $\left|\psi_{i}\right\rangle$. Using standard tensor analysis notation, this projection is

$$
E_{j_{1} \cdots j_{p}}^{i_{1} \cdots i_{p}}= \begin{cases}1 / n & \text { if } i_{1}=\cdots=i_{p} \text { and } j_{1}=\cdots=j_{p} \\ 0 & \text { otherwise. }\end{cases}
$$

For what follows we need to compute $\langle E, B\rangle$ for an arbitrary trace 1, Hermitian matrix, $B$. Using the decomposition in the previous section, we know that $B$ is the orthogonal sum of matrices $B(\alpha), \alpha \in \mathbb{Z}_{2}^{p}$, where $B(\alpha)=B_{1}(\alpha) \otimes \cdots \otimes B_{p}(\alpha)$, with $B_{j}(\alpha)=\frac{1}{n} I$ if $\alpha_{j}=0$ and $B_{j}(\alpha) \in \tau_{0}(n)$ if $\alpha_{j}=1$. Thus we only need to compute $\langle E, B(\alpha)\rangle$, since $\langle E, B\rangle$ is the sum of such quantities. 
Since $B(\alpha)$ is a product $(B(\alpha))_{t_{i} \ldots t_{p}}^{s_{1} \ldots s_{p}}=\left(B_{1}(\alpha)\right)_{t_{1}}^{s_{1}} \cdots\left(B_{p}(\alpha)\right)_{t_{p}}^{s_{p}}$. Again using the Einstein summation convention and using the fact $E_{j_{1} \cdots j_{p}}^{i_{1} \cdots i_{p}}=0$ unless $i_{1}=\cdots i_{p}$ and $j_{1}=\cdots j_{p}$, we obtain

$$
\begin{aligned}
\langle E, B(\alpha)\rangle & =E_{j_{1} \cdots j_{p}}^{i_{1} \cdots i_{p}}\left(B_{1}(\alpha)\right)_{i_{1}}^{j_{1}} \cdots\left(B_{p}(\alpha)\right)_{i_{p}}^{j_{p}} \\
& =E_{j \cdots j}^{i \cdots i}\left(B_{1}(\alpha)\right)_{i}^{j} \cdots\left(B_{p}(\alpha)\right)_{i}^{j}
\end{aligned}
$$

When $|\alpha|<p$ at least one of the $\alpha_{j}=0$ and so at least one of the $B_{j}(\alpha)=\frac{1}{n} I$. For this one we have $\left(B_{l}(\alpha)\right)_{i}^{j}=\left\{\begin{array}{c}0 \text { if } i \neq j \\ \frac{1}{n} \text { if } i=j\end{array}\right.$. Thus in this case (8) becomes

$$
\begin{aligned}
\langle E, B(\alpha)\rangle & =E_{i \cdots i}^{i \cdots i}\left(B_{1}(\alpha)\right)_{i}^{i} \cdots\left(B_{p}(\alpha)\right)_{i}^{i}, \\
& =\frac{1}{n}\left(B_{1}(\alpha)\right)_{i}^{i} \cdots\left(B_{p}(\alpha)\right)_{i}^{i} .
\end{aligned}
$$

Theorem 5 The maximally entangled state $E$ is in the set normal to $\Sigma$ at $\frac{1}{N} I$.

Proof:

What it means for $E$ to be in the set normal to $\Sigma$ at $\frac{1}{N} I$ is that the vector parallel to the line which connects $\frac{1}{N} I$ to $E$ is perpendicular to any vector tangent to $\Sigma$ at $\frac{1}{N} I$. We have seen above that these tangent vectors are of the form $\sum_{|\alpha|=1} B(\alpha)$. Hence we need to show that $\left\langle E-\frac{1}{N} I, \sum_{|\alpha|=1} B(\alpha)\right\rangle=0$. This follows because

$$
\left\langle E-\frac{1}{N} I, \sum_{|\alpha|=1} B(\alpha)\right\rangle=\sum_{|\alpha|=1}\left(\langle E, B(\alpha)\rangle-\frac{1}{N}\langle I, B(\alpha)\rangle\right.
$$

and all the terms on the right hand side of (10) are zero. Indeed the term $\frac{1}{N}\langle I, B(\alpha)\rangle=0$, since $B(\alpha)$ is trace 0 . And the $\langle E, B(\alpha)\rangle$ are 0 because $|\alpha|=1$. When $|\alpha|=1$, there is only one factor in $B(\alpha)$ which is not $\frac{1}{n} I$, and so $\langle E, B(\alpha)\rangle$ reduces to $\frac{1}{n^{p}}\left(B_{j}(\alpha)\right)_{i}^{i}$, with $B_{j}(\alpha)$ being the one factor which is not $\frac{1}{n} I$. But $\left(B_{j}(\alpha)\right)_{i}^{i}$ is the trace of $B_{j}(\alpha)$, which is 0 .

Because of this theorem, we know there is the possibility that the closest product state to $E$ is $\frac{1}{N} I$. We shall now show that is in fact the case for two particles, but not the case 
for more than two. First of all we note that the distance squared from $E$ to any other state $C$ is

$$
\langle E-C, E-C\rangle=\|E\|^{2}-2 \operatorname{Re}\langle E, C\rangle+\|C\|^{2} .
$$

When $C$ is the totally mixed state, $\frac{1}{N} I$, this reduces to $\|E\|^{2}-\frac{1}{N}$. We need to compare this with the distance from $E$ to a product state. Suppose $A$ is a product state. Then $A=\left(\frac{1}{n} I+R_{1}\right) \otimes \cdots \otimes\left(\frac{1}{n} I+R_{p}\right)$, where $R_{j} \in \tau_{0}(n)$. Expanding this expression for $A$, we get $A=\frac{1}{N} I+\sum_{k=1}^{p} \sum_{|\alpha|=k} B(\alpha)$, where for each $\alpha \in \mathbb{Z}_{2}^{p}$ we have $B(\alpha)=B_{1}(\alpha) \otimes \cdots \otimes B_{p}(\alpha)$, with $B_{j}(\alpha)=\frac{1}{n} I$ if $\alpha_{j}=0$ and $B_{j}(\alpha)=R_{j}$ if $\alpha_{j}=1$. Recall from above that the $B(\alpha)$ are mutually orthogonal and there are $\left(\begin{array}{l}p \\ k\end{array}\right)$ of them for each $k$. Set $B_{k}=\sum_{|\alpha|=k} B(\alpha)$. The $B_{k}$ are also mutually orthogonal. In terms of them, $A=\frac{1}{N} I+\sum_{k=1}^{p} B_{k}$.

Using the mutual orthogonality of the $B_{k}$, we find

$$
\begin{aligned}
\langle E, A\rangle & =\left\langle E, \frac{1}{N} I\right\rangle+\sum_{k=1}^{p}\left\langle E, B_{k}\right\rangle, \\
& =\frac{1}{N}+\sum_{k=1}^{p}\left\langle E, B_{k}\right\rangle .
\end{aligned}
$$

Similarly, $\|A\|^{2}=\frac{1}{N}+\sum_{k=1}^{p}\left\|B_{k}\right\|^{2}$. Hence it follows from (11) that the distance squared from $E$ to $A$ is

$$
\langle E-A, E-A\rangle=\|E\|^{2}-\frac{1}{N}-2 \operatorname{Re} \sum_{k=1}^{p}\left\langle E, B_{k}\right\rangle+\sum_{k=1}^{p}\left\|B_{k}\right\|^{2} .
$$

Recalling that the distance squared from $E$ to the totally mixed state is $\|E\|^{2}-\frac{1}{N}$, we see that the totally mixed state is the closest product state to $E$ if and only if $\sum_{k=1}^{p}\left(\left\|B_{k}\right\|^{2}-\right.$ $\left.2 \operatorname{Re}\left\langle E, B_{k}\right\rangle\right) \geq 0$ for all choices of $R_{1}, \ldots, R_{p} \in \tau_{0}(n)$ such that $A$ is a density matrix. Let us first compute $\left\langle E, B_{k}\right\rangle$. For $k<p$ we can use (9) to get

$$
\begin{aligned}
\left\langle E, B_{k}\right\rangle & =\sum_{|\alpha|=k}\langle E, B(\alpha)\rangle, \\
& =\sum_{|\alpha|=k} \frac{1}{n}\left(B_{1}(\alpha)\right)_{i}^{i} \cdots\left(B_{p}(\alpha)\right)_{i}^{i} .
\end{aligned}
$$


Suppose for $\alpha=\left(\alpha_{1}, \ldots, \alpha_{p}\right)$ that $\alpha_{j}=1$ for $j=m_{1}, \ldots, m_{k}$. Then the summand in (13) becomes $\frac{1}{n^{p-k+1}}\left(R_{m_{1}}\right)_{i}^{i} \cdots\left(R_{m_{k}}\right)_{i}^{i}$. Thus for $k<p$ we have

$$
\left\langle E, B_{k}\right\rangle=\frac{1}{n^{p+1-k}} \sum_{m_{1}<\cdots<m_{k}}\left(R_{m_{1}}\right)_{i}^{i} \cdots\left(R_{m_{k}}\right)_{i}^{i} .
$$

For $k=p$ we have $\left\langle E, B_{p}\right\rangle=E_{j \ldots j}^{i \ldots i}\left(R_{1}\right)_{i}^{j} \cdots\left(R_{p}\right)_{i}^{j}=\frac{1}{n} \sum_{i, j}\left(R_{1}\right)_{i}^{j} \cdots\left(R_{p}\right)_{i}^{j}$. Hence

$$
\begin{aligned}
2 \operatorname{Re} \sum_{k=1}^{p}\left\langle E, B_{k}\right\rangle= & \frac{2}{n^{p+1}} \sum_{k=1}^{p} \sum_{m_{1}<\cdots<m_{k}}\left(n R_{m_{1}}\right)_{i}^{i} \cdots\left(n R_{m_{k}}\right)_{i}^{i} \\
& +\frac{2}{n} \operatorname{Re} \sum_{i \neq j}\left(R_{1}\right)_{i}^{j} \cdots\left(R_{p}\right)_{i}^{j} .
\end{aligned}
$$

Dispensing with the Einstein summation notation from here on out, we can rewrite (15) as

$$
\begin{aligned}
\left\langle E, B_{k}\right\rangle= & \left.\frac{2}{n^{p+1}}\left(\sum_{i=1}^{n}\left(\prod_{l=1}^{p}\left(1+n\left(R_{l}\right)_{i}^{i}\right)\right)-1\right)\right)+\frac{2}{n} \operatorname{Re} \sum_{i \neq j}\left(R_{1}\right)_{i}^{j} \cdots\left(R_{p}\right)_{i}^{j} .
\end{aligned}
$$

We also need to compute $\left\|B_{k}\right\|^{2}$. Fortunately, this is quite simple since the $B(\alpha)$ are orthogonal. In particular, we have for all $k$ that

$$
\begin{aligned}
\left\|B_{k}\right\|^{2} & =\sum_{|\alpha|=k}\|B(\alpha)\|^{2}=\sum_{|\alpha|=k}\left\|B_{1}(\alpha)\right\|^{2} \cdots\left\|B_{p}(\alpha)\right\|^{2}, \\
& =\frac{1}{n^{p-k}} \sum_{m_{1}<\cdots<m_{k}}\left\|R_{m_{1}}\right\|^{2} \cdots\left\|R_{m_{k}}\right\|^{2} .
\end{aligned}
$$

Thus

$$
\begin{aligned}
\sum_{k=1}^{p}\left\|B_{k}\right\|^{2} & =\frac{1}{n^{p}} \sum_{k=1}^{p} \sum_{m_{1}<\cdots<m_{k}} n\left\|R_{m_{1}}\right\|^{2} \cdots n\left\|R_{m_{k}}\right\|^{2}, \\
& =\frac{1}{n^{p}}\left(\prod_{l=1}^{p}\left(1+n\left\|R_{l}\right\|^{2}\right)-1\right) .
\end{aligned}
$$

We can now rephrase our question as follows: is there a choice of $R_{j} \in \tau_{0}(n)$ such that $A=\left(\frac{1}{n} I+R_{1}\right) \cdots\left(\frac{1}{n} I+R_{p}\right)$ is a density matrix and

$$
\begin{aligned}
& \frac{1}{n^{p}}\left(\prod_{l=1}^{p}\left(1+n\left\|R_{l}\right\|^{2}\right)-1\right) \\
& \left.-\frac{2}{n^{p+1}}\left(\sum_{i=1}^{n}\left(\prod_{l=1}^{p}\left(1+n\left(R_{l}\right)_{i}^{i}\right)\right)-1\right)\right)-\frac{2}{n} \operatorname{Re} \sum_{i \neq j}\left(R_{1}\right)_{i}^{j} \cdots\left(R_{p}\right)_{i}^{j}
\end{aligned}
$$


is negative. If so, then that product state is closer to the maximally entangled state than the totally mixed one. If not, then the totally mixed state is the closest product state to the maximally entangled one.

Theorem 6 For a quantum system modelled on $\mathbb{C}^{n} \otimes \mathbb{C}^{n}$ the closest product state to a maximally entangled state is the totally mixed one. Because of this there are no product states within $\sqrt{\frac{N-1}{N}}$, where $N=n^{2}$, of a maximally entangled state. For quantum systems with more than two particles, the totally mixed state is not the closest product state to a maximally entangled state.

Proof:

Let us first consider the case of two particles. Thus $p=2$ in (19) and so it reduces to

$$
\begin{aligned}
& \frac{1}{n^{2}}\left(1+n\left\|R_{1}\right\|^{2}\right)\left(1+n\left\|R_{2}\right\|^{2}\right)-\frac{1}{n^{2}} \\
& -\frac{2}{n^{3}} \sum_{i=1}^{n}\left(\left(1+n\left(R_{1}\right)_{i}^{i}\right)\left(1+n\left(R_{2}\right)_{i}^{i}\right)-1\right) \\
& -\frac{2}{n} \operatorname{Re} \sum_{i \neq j}\left(R_{1}\right)_{i}^{j}\left(R_{2}\right)_{i}^{j} .
\end{aligned}
$$

Expanding the first two terms, cancelling, regrouping and using the fact the $R_{l}$ are Hermitian and so $\left(R_{2}\right)_{i}^{j}={\overline{\left(R_{2}\right)}}_{j}^{i}$ we see that $\left.(\sqrt[20)]{ }\right)$ equals

$$
\begin{aligned}
& \frac{1}{n}\left(\left\|R_{1}\right\|^{2}+\left\|R_{2}\right\|^{2}-2\left[\left(R_{1}\right)_{i}^{i}\left(R_{2}\right)_{i}^{i}+\operatorname{Re} \sum_{i \neq j}\left(R_{1}\right)_{i}^{j}{\overline{\left(R_{2}\right.}}_{j}^{i}\right]\right) \\
& +\left\|R_{1}\right\|^{2}\left\|R_{2}\right\|^{2}-\frac{2}{n^{2}}\left(\left(R_{1}\right)_{i}^{i}+\left(R_{2}\right)_{i}^{i}\right) .
\end{aligned}
$$

Since the $R_{l}$ are trace 0 , the last term in this expression is 0 . On the other hand the first term is $\frac{1}{n}\left\langle R_{1}-\overline{R_{2}}, R_{1}-\overline{R_{2}}\right\rangle$, which is greater than or equal to zero, with equality only if $R_{1}=R_{2}=0$. Since the same is true for the middle term in (20), we have proved that $\frac{1}{N} I$ is the product state closest to $E$ in the bipartite case.

Now suppose $p \geq 3$ and take all the $R_{l}$ to be the matrix $R$ with diagonal elements:

$$
R_{i}^{j}= \begin{cases}1 / 2 n & \text { if } i=j=1 \\ -1 / 2 n & \text { if } i=j=2 \\ 0 & \text { otherwise. }\end{cases}
$$


When (19) has this $R$ substituted into it, it becomes

$$
\frac{1}{n^{p}}\left(\left(1+\frac{1}{2 n}\right)^{p}+\frac{4}{n}-1-\frac{2}{n}\left(\left(\frac{3}{2}\right)^{p}+\left(\frac{1}{2}\right)^{p}\right)\right)
$$

For $p=3$, this reduces to $8 n^{3}\left(-12 n^{2}+6 n+1\right)$, which is negative for $n \geq \dot{2}$. Since for such $n$ the quantity $\left(1+\frac{1}{2 n}\right)$ is less than $\frac{3}{2}$, for fixed $n \geq 2$, the expression $\left(1+\frac{1}{2 n}\right)^{p}+\frac{4}{n}-$ $1-\frac{2}{n}\left(\left(\frac{3}{2}\right)^{p}+\left(\frac{3}{2}\right)^{p}\right)$ decreases with increasing $p$ and so is negative for all $p \geq 3$.

In conclusion, the geometry of states was studied. Prior results by Sanpera, Vidal and Tarrach were extended and several new results were presented. These results are useful in the characterization of entangled states, which has been a difficult problem due to the complicated structure of the entangled space.

Acknowledgement: The authors acknowledge the Office of Naval Research for the support of this work.

\section{APPENDIX}

In this Appendix an algorithm is presented to find the closest product state to an arbitary state. It was used, for example, to initially find that the closest product state to the maximally entangled state is the maximally mixed state. The algorithm converges very rapidly (often within a few iterations) for modest $N$, where $N=n^{2}$.

Given a density matrix $C \in \mathbb{C}^{n} \otimes \mathbb{C}^{n}$, consider the problem of finding

$$
\arg \min _{A, B}\|C-A \otimes B\|
$$

where $A, B \in \mathbb{C}^{n}$ are density matrices. An outline of the algorithm is first provided, and later the details on the computation of $A_{o}$ and $B_{o}$ are given.

\section{A. Algorithm}

1. Set $B=I_{n}$. 
2. Given $B$, find $A_{o}$ as computed in Subsection B.

- Set $A=A_{o}+\frac{1}{n}\left(1-\operatorname{Tr}\left(A_{o}\right)\right) I_{n}$

- Check if $A$ is positive semi-definite (psd). If yes, then $A$ satisfies a Lagrange Multiplier equation (see (23) and discussion) skip to Step 3. If no, an $A$ that satisfies a Lagrange Multiplier equation (see (30) and discussion) can be found as follows:

- Compute the eigenvalues of $A_{o}$, represented by $\lambda_{1} \geq \lambda_{2} \cdots \geq \lambda_{n}$ and let $\Phi_{1}, \Phi_{2}, \cdots, \Phi_{n}$ be corresponding eigenvectors.

- Set $M=\arg \min _{m}\left\{m \mid(n-1) \lambda_{j}+\lambda_{m}+1-\operatorname{Tr}\left(A_{o}\right) \geq 0, j=1,2, \cdots, n ; j \neq m\right\}$.

- For $m=1,2, \cdots, M$, compute $A_{m}=A_{o}+\frac{1}{n-1}\left(1-\operatorname{Tr}\left(A_{o}\right)+\lambda_{m}\right) I_{n}-\frac{1}{n-1}(1-$ $\left.\operatorname{Tr}\left(A_{o}\right)+n \lambda_{m}\right) \Phi_{m} \Phi_{m}^{\prime}$

- Set $A=\arg \min _{A_{m}}\left\|C-A_{m} \otimes B\right\|_{2}$

3. Given $A$ find $B_{o}$. This is accomplished by considering the element transform $\mathrm{T}$ such that $\mathrm{T}(A \otimes B)=B \otimes A$. Setting $\tilde{C}=\mathrm{T}(C)$, it is seen that $\|C-A \otimes B\|=\|\tilde{C}-B \otimes A\|$. Then apply the methodology as in Steps 1. and 2. above with the appropriate changes to the transformed problem.

4. Check $\|C-A \otimes B\|$ for convergence. If no, go to Step 2.

\section{B. Solution for $A_{o}$ and $B_{o}$}

Finding $A_{o}$ is equivalent to finding $a_{i j}(i, j=1,2, \cdots, n)$ such that

$$
a_{i j}=\arg \min _{a_{i j}}\left\|C_{i j}-a_{i j} B\right\|
$$

Now

$$
\left\|C_{i j}-a_{i j} B\right\|^{2}=\operatorname{Tr}\left\{\left(C_{i j}-a_{i j} B\right)\left(C_{i j}-a_{i j} B\right)^{\prime}\right\}
$$

where ' denotes conjugate transpose. The above is minimized when 


$$
\begin{aligned}
& a_{i j}^{o}=\frac{\operatorname{Tr}\left(C_{i j} B^{\prime}\right)}{\|B\|^{2}} \quad i \neq j, \\
& a_{i i}^{o}=\frac{1}{2} \frac{\operatorname{Tr}\left(C_{i i} B^{\prime}\right)+\operatorname{Tr}\left(B C_{i i}^{\prime}\right)}{\|B\|^{2}} .
\end{aligned}
$$

Finding $B_{o}$ can be easily accomplished with the same result as above, by considering the element transform $\mathrm{T}$ such that $\mathrm{T}(A \otimes B)=B \otimes A$. Setting $\tilde{C}=\mathrm{T}(C)$, it is seen that

$$
\|C-A \otimes B\|=\|\tilde{C}-B \otimes A\|
$$

and the solution for $b_{i j}^{o}$ is by symmetry with (22) given by

$$
\begin{aligned}
b_{i j}^{o} & =\frac{\operatorname{Tr}\left(\tilde{C}_{i j} A^{\prime}\right)}{\|A\|^{2}} \quad i \neq j, \\
b_{i i}^{o} & =\frac{1}{2} \frac{\operatorname{Tr}\left(\tilde{C}_{i i} A^{\prime}\right)+\operatorname{Tr}\left(A \tilde{C}_{i i}^{\prime}\right)}{\|A\|^{2}} .
\end{aligned}
$$

Remark The solution to $A$ without the constraint $\operatorname{Tr}(A)=1$ for

$$
\arg \min _{A}\|C-A \otimes B\|
$$

where $B, C$ are psd Hermitian (psdh) matrices, can be shown to be psdh. Hence both $A_{o}$ and $B_{o}$ are always psd.

Consider now the solution to $A$ with $\operatorname{Tr}(A)=1$ for

$$
\arg \min _{A}\|C-A \otimes B\|
$$

where $B, C$ are given density matrices. The Lagrange multiplier solution to

$$
\nabla_{A}\left\{\|C-A \otimes B\|_{2}+\gamma \operatorname{Tr}(A)\right\}=0
$$

can be shown to have the form $A=A_{o}+\tilde{\gamma} I_{n}$, where $\nabla_{A}$ is the gradient, $\nabla_{A} \triangleq \frac{\partial}{\partial A}$. Substituting this form in the constraint equation, $\operatorname{Tr}(A)=1$ gives $\tilde{\gamma}=1-\operatorname{Tr}\left(A_{o}\right)$. Since this is a convex minimization problem, an interior point solution to Lagrange multiplier gradient equations is guaranteed to be the minimum. If $A$ is $\operatorname{psd}$ ( $A$ is Hermitian), then we will use this solution in the algorithm, although strictly speaking, it should be checked to see if it is an interior point. 
The solution to $A$ with $\operatorname{Tr}(A)=1$ for

$$
\arg \min _{A \in \operatorname{psdh}}\|C-A \otimes B\|
$$

where $B, C$ are density matrices is not as straightforward if $A=A_{o}+\left(1-\operatorname{Tr}\left(A_{o}\right)\right) I_{n}$ is not psd. In this case, we use the additional constraints $x^{\prime} A x \geq 0$ and $x^{\prime} x=1$. The minimization problem is no longer convex. However, if the minimum is an interior point, then it necessarily satisfies the Lagrange multiplier gradient equations. As $A=A_{o}+\left(1-\operatorname{Tr}\left(A_{o}\right)\right) I_{n}$ is not psd but still minimizes $\|C-A \otimes B\|_{2}$, then the solution lies on the boundary of the constraint $x^{\prime} A x \geq 0$, i.e. $A$ is singular, hence $x^{\prime} A x=0$. Now, the Lagrange multiplier problem becomes the solution to:

$$
\begin{aligned}
& \nabla_{A}\left\{\|C-A \otimes B\|_{2}+\gamma_{1} \operatorname{Tr}(A)+\gamma_{2} x^{\prime} A x+\gamma_{3} x^{\prime} x\right\}=0 \\
& \nabla_{x}\left\{\|C-A \otimes B\|_{2}+\gamma_{1} \operatorname{Tr}(A)+\gamma_{2} x^{\prime} A x+\gamma_{3} x^{\prime} x\right\}=0 .
\end{aligned}
$$

Eqn (25) above merely shows that $x$ is an eigenvector of $A$. Equation (24) imples that $A$ has the following form:

$$
A=A_{o}+\tilde{\gamma}_{1} I_{n}+\tilde{\gamma}_{2} x x^{\prime}
$$

Now, $\tilde{\gamma_{1}}, \tilde{\gamma}_{2}$ must satisfy constraint equations:

$$
\operatorname{Tr}(A)=1=\operatorname{Tr}\left(A_{o}\right)+n \tilde{\gamma_{1}}+\tilde{\gamma_{2}}
$$

and

$$
\begin{aligned}
0 & =A x \\
& =A_{o} x+\tilde{\gamma_{1}} x+\tilde{\gamma_{2}} x \\
& =\left(A_{o}+\left(\tilde{\gamma_{1}}+\tilde{\gamma_{2}}\right) I_{n}\right) x
\end{aligned}
$$

Equation (27) implies that $x^{\prime}$ is an eigenvector of $A_{o}$. Denote the eigenvalues of $A_{o}$ by $\lambda_{1} \geq \lambda_{2} \cdots \geq \lambda_{n}$ and $\Phi_{1}, \Phi_{2}, \cdots, \Phi_{n}$ the corresponding eigenvectors. Let $x=\Phi_{m}$, then $A x=0$ implies 


$$
\tilde{\gamma_{1}}+\tilde{\gamma_{2}}=-\lambda_{m}\left(A_{o}\right)
$$

Using (27) and (28) we can show

$$
A=A_{o}+\frac{1}{n-1}\left(1-\operatorname{Tr}\left(A_{o}\right)+\lambda_{m}\right) I_{n}-\frac{1}{n-1}\left(1-\operatorname{Tr}\left(A_{o}\right)+n \lambda_{m}\right) \Phi_{m} \Phi_{m}^{\prime} .
$$

We can show using (29) that $x^{\prime} A x=\Phi_{m}^{\prime} A \Phi_{m}=0$. It would appear, we have $n$ possible candidates for the solution of $A$ : each solution would be based on using a different $\Phi_{m}$ in (29). However, we still must ensure that $A$ is psd. The solutions for $A$ are Hermitian.

In order for $A$ to be psd, we required that the eigenvalues of $A$ (denoted by $\gamma_{j}, j=$ $1,2, \cdots, n)$ to satisfy $\gamma_{j} \geq 0$. Now, we can show if $x=\Phi_{m}$ then using (29) the following $n-1$ conditions must be satisfied:

$$
(n-1) \gamma_{j}=(n-1) \lambda_{j}+\lambda_{m}+1-\operatorname{Tr}\left(A_{o}\right) \geq 0, \text { for } j=1,2, \cdots, n, j \neq m .
$$

Note $\lambda_{1}=\max _{j} \lambda_{j}$. If the minimum occurs at an interior point then we are guaranteed that a solution for $A$ must exist and

$$
(n-1) \lambda_{j}+\lambda_{1}+1-\operatorname{Tr}\left(A_{o}\right) \geq(j-1) \lambda_{j}+\lambda_{m}+1-\operatorname{Tr}\left(A_{o}\right)
$$

for all $j, m$. Therefore

$$
(n-1) \lambda_{j}+\lambda_{1}+1-\operatorname{Tr}\left(A_{o}\right) \geq 0
$$

for all $j$. Thus we will successively check the largest eigenvalues of $A_{o}$ and see which satisfy (30) (again $\lambda_{1}$ must satisfy (30) if an interior point minimum exists). The solution for $A_{o}$ can be checked numerically to see if it is an interior point minimum. 


\section{REFERENCES}

[1] A.Einstein, B.Podolsky, N.Rosen, Phys.Rev. 47 (1935).

[2] J.S.Bell, Rev. Mod. Phys. 38 (1966) 447; and Physics I (1964) 195 .

[3] R.Omnes, The Interpretation of Quantum Mechanics, Princeton University Press (1994); and Understanding Quantum Mechanics, Princeton University Press (1999).

[4] C.H.Bennett, G.Brassard, C.Crepeau, R.Josza, A.Peres, and W.K.Wooters, Phys. Rev. Lett. 70 (1993) 1895.

[5] R.F.Werner, All Teleportation and Dense Coding Schemes, quant-ph/0003070.

[6] P.Shor and J.Preskill, Phys.Rev.Lett. 85 (2000) 441.

[7] W.T.Butler, et.al., Phys. Rev. Lett. 81 (1998)3283.

[8] A.Peres, Phys. Rev. Lett 77 (1996) 1413.

[9] M.Horodecki, P.Horodecki, and R.Horodecki, Separability of n-Particle Mixed States: Necessary and Sufficient Conditions in Terms of Linear Maps, quant-ph/0006071.

[10] Shengjun Wu and Yongde Zhang, A Necessary and Sufficient Criterion of Separability for Multiparticle Quantum States, quant-ph/0006058.

[11] K.Audennaert, F. Verstraete, B.De Moor, Variational Characterisations of Separability and Entanglement of Formation, quant-ph/0006128.

[12] A.Sanpera, R.Tarrach, and G. Vidal, Phys.Rev A 58 ( 1998) 826.

[13] R.Lockhart, Optimal Ensemble Length of Mixed Separable States, to appear Jour. Math.Phys. 